\title{
Vortex-antivortex nucleation in magnetically nanotextured superconductors: Magnetic-field-driven and thermal scenarios
}

\author{
M. V. Milošević and F. M. Peeter:* \\ Departement Fysica, Universiteit Antwerpen (Campus Middelheim), \\ Groenenborgerlaan 171, B-2020 Antwerpen, Belgium
}

(Dated: June 29, 2018)

\begin{abstract}
Within the Ginzburg-Landau formalism, we predict two novel mechanisms of vortex-antivortex nucleation in a magnetically nanostructured superconductor. Although counterintuitive, nucleation of vortex-antivortex pairs can be activated in a superconducting (SC) film covered by arrays of submicron ferromagnets (FMs) when exposed to an external homogeneous magnetic field. In another scenario, we predict the thermal induction of vortex-antivortex configurations in SC/FM samples. This phenomenon leads to a new type of Little-Parks oscillations of the FM magnetizationtemperature phase boundary of the superconducting film.
\end{abstract}

PACS numbers: 74.78.-w, 74.78.Na, 74.25.Dw, 74.25.Qt.

Over the years, increased attention has been paid to experimental and theoretical studies of vortex-antivortex (VAV) phenomena in diverse systems. For example, the analogous process of electron-positron pair creation and annihilation is well known in quantum electrodynamics 1]. The appearance of VAV structures has also been revealed in elastic waves in dusty plasmas 2], Bose-Einstein condensates [3] , and 3D connected superfluid films [4].

In the last decade, thermally activated VAV nucleation has been extensively studied in two-dimensional superconducting (SC) films [5] and Josephson junctions [6]. The Berezinskii-Kosterlitz-Thouless (BKT) transition takes place at finite temperature, below which the vortex-antivortex pairs are bound and the sample resistance is zero [7]. In another scenario, a VAV pair in SC film may be created (or depinned) by a short lived local hot spot caused by the absorption of a single photon [8]. Recently, vortex-antivortex structures were predicted in mesoscopic superconducting polygons in homogeneous magnetic field [9]. Those VAV states are imposed by the symmetry of the sample, close to the nucleation line $T_{c}(H)$. However, they become unstable deeper in the SC state, except for effective type I superconductors [10].

Intuitively, states containing vortex-antivortex pairs are relevant to superconductors exposed to an inhomogeneous magnetic field. In this respect, SC films with ferromagnetic (FM) structures on top have been studied, and broken symmetry VAV states [1], VAV molecules and VAV lattices of different geometries 12] have been predicted. The broader aspect of the vortex-antivortex phenomena becomes apparent as the found structures resemble the ones of electron dimples on the surface of liquid helium, colloidal suspensions, dusty particles in complex plasmas, and ionic or molecular crystals.

In this Letter, we report two new mechanisms of vortex-antivortex nucleation in SC films under regular arrays of nanoengineered FM-dots (see Fig. (1). Such hybrid structures have been realized experimentally 13, 14]. However, these and following works were focused on commensurability effects with magnetic pinning arrays, the consequent critical current enhancement and the so-called field-induced superconductivity, crucial for possible applications. The main objective of this Letter is to explore the dynamical behavior of SC/FM samples when exposed to thermal excitations and/or an additional homogeneous magnetic field.

Our theoretical formalism relies upon the timedependent Ginzburg-Landau (GL) equations for the order parameter $\Psi$ and the vector potential A [15], supplemented by a random force in order to include the thermodynamic fluctuations in $\Psi(\mathbf{r}, t)$. In dimensionless form, when keeping the temperature dependence explicitly, the
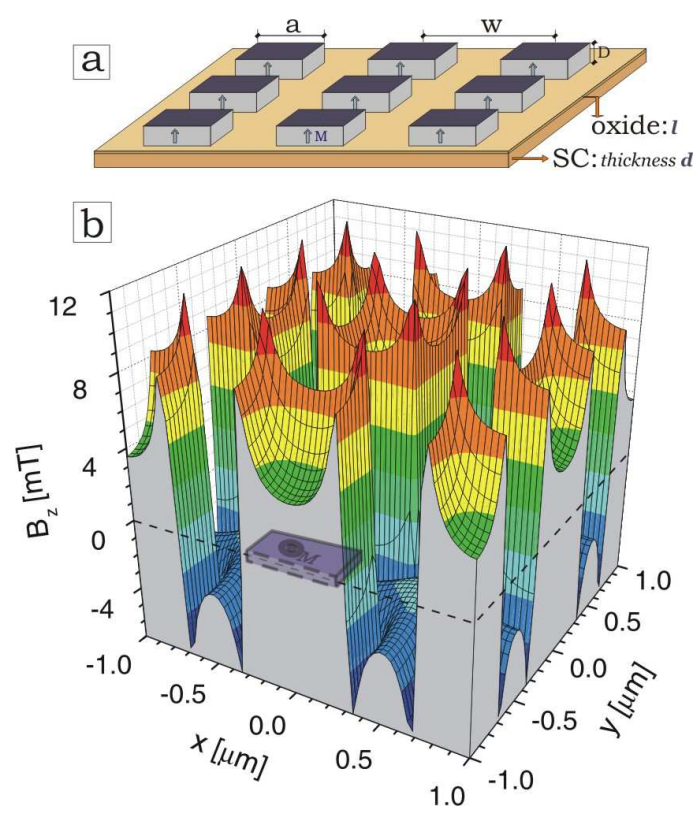

FIG. 1: (a) A regular array of square magnetic dots (MDs) on top of a superconducting (SC) film (separated by an oxide layer); (b) the MDs-magnetic field profile in the SC plane $(a=$ $0.6 \mu \mathrm{m}, W=1 \mu \mathrm{m}, D=25 \mathrm{~nm}$, and bulk Co magnetization). 
GL equations become

$$
\frac{\partial \Psi}{\partial t}=-\frac{1}{\zeta}\left[(-i \nabla-\mathbf{A})^{2} \Psi+\Psi\left(1-T-|\Psi|^{2}\right)\right]+f(\mathbf{r}, t),
$$

$$
\frac{\partial \mathbf{A}}{\partial t}=\operatorname{Re}\left[\Psi^{*}\left(\frac{\nabla}{i}-\mathbf{A}\right) \Psi\right]-\kappa^{2} \nabla \times \nabla \times \mathbf{A},
$$

where distances are expressed in units of $\xi(0), \mathbf{A}$ in $H_{c 2}(0) \xi(0)$, time in $t_{0}=\pi \hbar / 96 k_{B} T_{c}$, temperature is scaled by $T_{c}$, and $\Psi$ is normalized by its equilibrium value in the absence of magnetic field. The relaxation constant $\zeta=12$ is taken from the microscopic theory [16]. $f(\mathbf{r}, t)$ is the dimensionless Langevin thermal noise, uncorrelated in space and time $\left\langle f^{*}(\mathbf{r}, t) f\left(\mathbf{r}^{\prime}, t^{\prime}\right)\right\rangle=$ $\frac{4 \pi}{\zeta} E_{0} T \delta\left(\mathbf{r}-\mathbf{r}^{\prime}\right) \delta\left(t-t^{\prime}\right)$, where $E_{0}=2 k_{B} T_{c} / H_{c}(0)^{2} \xi(0)^{3}$ is the ratio of the thermal energy to the free energy of a vortex. The random force $f_{i}(t)$ at site $i$ is independently selected from a Gaussian distribution with a zero mean.

For thin superconductors $(d<\xi, \lambda)$, Eqs. (12) may be averaged over the SC thickness. The lateral periodicity of the SC film (and the magnetic structures on top of it) is included in our formalism through the periodic boundary conditions $\mathbf{A}\left(\mathbf{r}+\mathbf{b}_{i}\right)=\mathbf{A}(\mathbf{r})+\nabla \eta_{i}(\mathbf{r})$, and $\Psi\left(\mathbf{r}+\mathbf{b}_{i}\right)=\Psi \exp \left(2 \pi i \eta_{i}(\mathbf{r}) / \Phi_{0}\right)$, where $\mathbf{b}_{i=x, y}$ are the periodicity vectors, and $\eta_{i}$ is the gauge potential. The choice of gauge and details of the numerical approach are explained in Ref. 12]. For given magnetization of the ferromagnets $M$, we explore the behavior of the superconducting state of the film starting the calculation from randomly generated $(\psi, \mathbf{A})$. In addition, field-cooled and zero-field-cooled regimes are simulated when Meissner state $(\Psi \approx 1)$ or the normal state $(\Psi \approx 0)$ are used as initial state. The ground state is then determined by comparing Gibbs free energy $(\mathcal{G})$ of all found states.

Influence of temperature. The Little-Parks (LP) effect [17, i.e. oscillations of the critical temperature $T_{c}$ with change in the external magnetic field $H_{\text {ext }}$, is in general associated with transitions between states with different vorticity in multiply connected superconducting samples. In this paper we focus on another possibility for creating multiquantum vortex states: nucleation of the superconducting order parameter in a hybrid system consisting of a thin superconducting film and an array of square magnetic nanodots (MDs) as a function of MD-magnetization $M$ (Fig. 1(a)). Provided that the thickness of the SC film is small $(d<\xi)$, the temperature of the $\mathrm{S} / \mathrm{N}$ transition as well as the structure of the superconducting nuclei are mainly determined by a $2 \mathrm{D}$ distribution of the MDmagnetic field (Fig. 1(b)). This magnetic field landscape is inhomogeneous, with one crucial property - its total flux through an infinite underlying plane equals zero. As a result, vortices cannot nucleate without corresponding antivortices, keeping the total vorticity at zero value.

The calculated $M-T$ phase diagram is shown in Fig. 2 for the typical values of the parameters corresponding to $\mathrm{Pb}$ or $\mathrm{Nb}$ films. The solid line indicates the superconducting/normal state $(\mathrm{S} / \mathrm{N})$ boundary $\left(|\psi|_{\max }^{2}<10^{-5}\right.$ denotes the normal state). The dotted curves bound the stability regions of the vortex-antivortex configurations deep in the SC state and dashed lines denote the transitions in the ground-state. As one can see, for example for $T / T_{c}=0.91$, increasing MD-magnetization leads to the appearance of more vortex-antivortex pairs $\left(N_{v-a v}\right.$ is the number of VAV pairs per MD), which arrange themselves in lattices (see Ref. [12]).

The crucial result here is the LP-type of oscillations in the $\mathrm{S} / \mathrm{N}$ boundary. However, significant differences are present, as compared to the usual LP-effect. Firstly, instead of the external field, the magnetization of the MDs is changed. As a result, the cusps in the phase boundary correspond now to the nucleation of vortex-antivortex pairs, not to individual vortices. Secondly, the total vorticity is always zero, and the analogue of the winding number in our system is the number of (anti)vortices (between) under the magnetic squares. For the same reason, the position of cusps in the boundary will be determined by the positive magnetic flux $\Phi^{+}$under each magnet. However, flux quantization does not have to be fulfilled, contrary to the conventional LP-effect. Actually, $\Phi^{+}$needed for the appearance of the first VAV pair is larger than the quantized $\Delta \Phi^{+}$necessary for the nucleation of the following pairs 12]. This LP-behavior of the $\mathrm{S} / \mathrm{N}$ boundary with variable period of the oscillations is the first such prediction for $S C$ films. The standard $\mathrm{H}_{\text {ext }}$ - $\mathrm{T}$ boundary for SC films does not exhibit any oscillations 14] $\left(\mathrm{H}_{c r}(\mathrm{~T})=\Phi_{0} / 2 \pi \xi(\mathrm{T})^{2}\right)$. As explained in Ref. [12], vortex-antivortex configurations created by

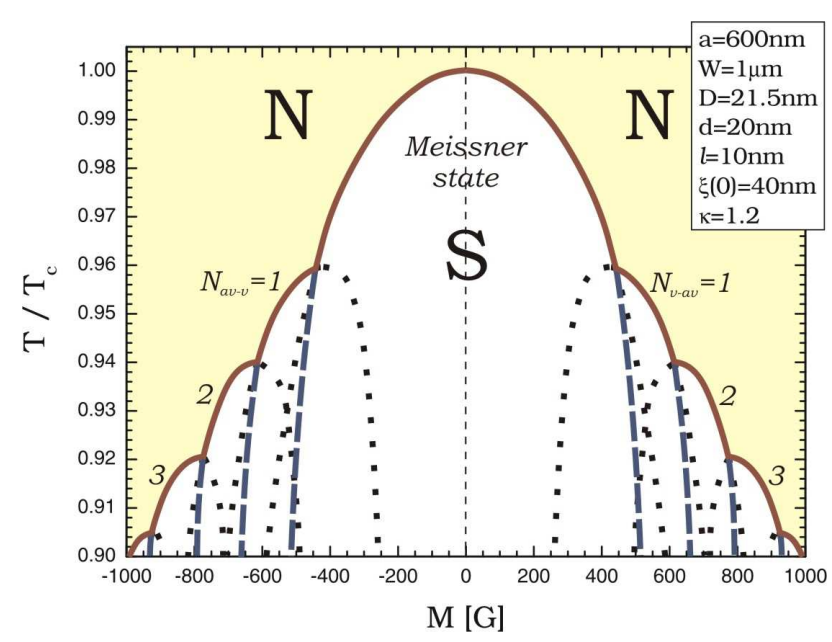

FIG. 2: Temperature versus MD-magnetization phase diagram. The solid line is the superconducting/normal state boundary. Dotted curves show the stability regions of different vortex-antivortex configurations while dashed lines denote transitions in the ground-state. For $M>0$, vortices are under the MDs and antivortices between them (and vice versa). $N_{v-a v}\left(N_{a v-v}\right)$ is the number of created pairs per MD. 


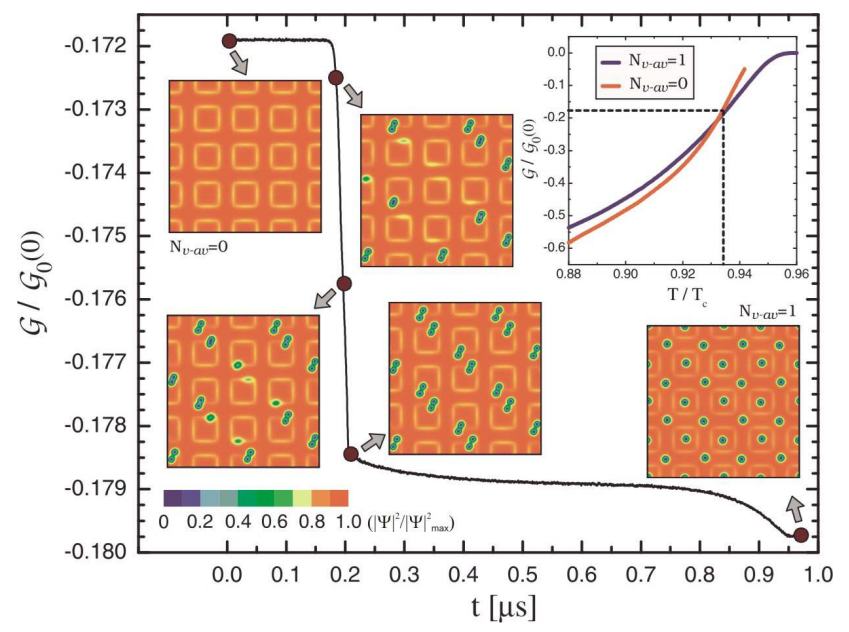

FIG. 3: The $N_{v-a v}=0-1$ transition at $T / T_{c}=0.935$ for $M=480 \mathrm{G}\left(\mathcal{G}_{0}(0)=H_{c}(0)^{2} V / 8 \pi\right.$, inset shows the energy of the states as a function of temperature). The $|\psi|^{2}$-density plots illustrate spontaneous vortex-antivortex nucleation due to thermal fluctuations.

regular MD-arrays are very compact and stable. Therefore, standard transport measurements could confirm the predicted shape of the phase boundary, through the measurements of the resistance variations with changing magnetic moment of each MD (or alternatively current in a loop) at a fixed temperature.

On the other hand, changing temperature for a fixed MD-magnetization reveals another fascinating phenomenon. Namely, in a zero-field cooled regime, the system relaxes in a vortex state which is not necessarily the ground-state. If then temperature is gradually changed (and thermal noise negligible), the vorticity remains the same, due to finite energy barrier (analogous to the Bean-Livingston one in finite samples), as long as the given state is stable (see inset in Fig. 33). However, increasing temperature strengthens thermal fluctuations and lowers the barrier. As shown in Fig. [3] for $M=480 \mathrm{G}$, the initial $N_{v-a v}=0$ state transits into $N_{v-a v}=1$ as soon as its energy becomes higher with increasing $T$. The energy barrier at particular MDs can be overcome by the random thermal fluctuations, and vortex-antivortex (VAV) pairs nucleate under the MD-edge where current is maximal. Each VAV pair then causes VAV nucleation at the neighboring site, due to the local supercurrent enhancement (see next paragraph). Eventually, each MD bounds one VAV pair and the system gradually relaxes into the ground-state $N_{v-a v}=1$ lattice. Therefore, vortex-antivortex pairs can be induced in SC films by increasing temperature. Although the nucleation of all VAV pairs takes less than $30 \mathrm{~ns}$, the whole $N_{v-a v}=0 \rightarrow 1$ transition takes a relatively long time $t \approx 1 \mu \mathrm{s}$. This facilitates the experimental observation of the thermally induced VAV pairs, by e.g. Scanning Hall Probe, Magnetic Force, or Lorentz Microscopy.
Influence of applied homogeneous field. If our sample is exposed to an additional homogeneous magnetic field $H_{\text {ext }}$, we expect the symmetry breaking of the phase boundary of Fig. 2 due to the interaction between the MD-induced VAV pairs and external flux lines. Namely, MDs attract the added vortices for $\mathbf{M} \| \mathbf{H}_{e x t}$, and vice versa [13. In what follows, we will focus on the part of the M-T diagram where no MD-induced VAV pairs exist, namely for $1>T / T_{c}>0.96$. At these temperatures, increasing $M$ continuously suppresses the order parameter under MDs (see Fig. 团 inset (a)), until the transition to the normal state (red curve in Fig. 4). Although critical conditions are met, no vortex-antivortex pairs can be induced in the underlying superconductor, even in the case of very strong magnets. Namely, vortices and antivortices cannot be adequately separated and stabilized since magnetic lattice is too dense compared to $\xi(\mathrm{T})[12]$.

However, the very same SC/FM sample demonstrates different behavior when exposed to homogeneous magnetic field $\left(\mathbf{H}_{\text {ext }} \| \mathbf{M}\right)$. We applied $H_{\text {ext }}=0.646 \mathrm{mT}$, creating five flux quanta piercing through the $4 \mathrm{x} 4$ unit cell. For weaker MDs, conventional pinning phenomena are observed, as external vortices are pinned by MDs (see [13]), in a specific regular configuration (see Fig. [4 inset (b)). However, for higher magnetization, two fascinating phenomena occur: (i) the energy of the sample exposed to $H_{\text {ext }}$ becomes lower than the one in $H_{\text {ext }}=0$ case, and (ii) VAV pairs nucleate and the most favorable vortex state consists of 9 vortices under MDs interconnected by

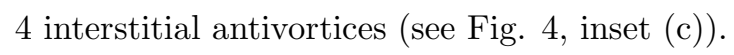

The explanation for these findings lies in the supercurrents. The vortex nucleation in superconductors is gov-

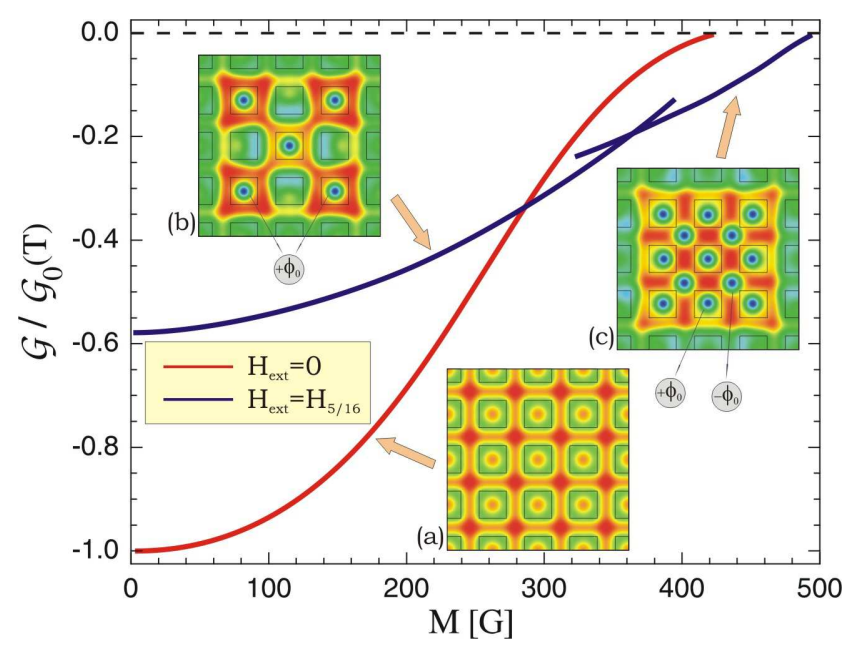

FIG. 4: The Gibbs free energy as a function of MDmagnetization at $T / T_{c}=0.97$, in the case of no applied homogeneous magnetic field $H_{\text {ext }}=0$ and rational $H_{e x t}=$ $H_{5 / 16}$ field. The insets show the Cooper-pair density plots: (a) Meissner state, (b) 5 pinned vortices, and (c) vortexantivortex configuration with total vorticity 5 . 


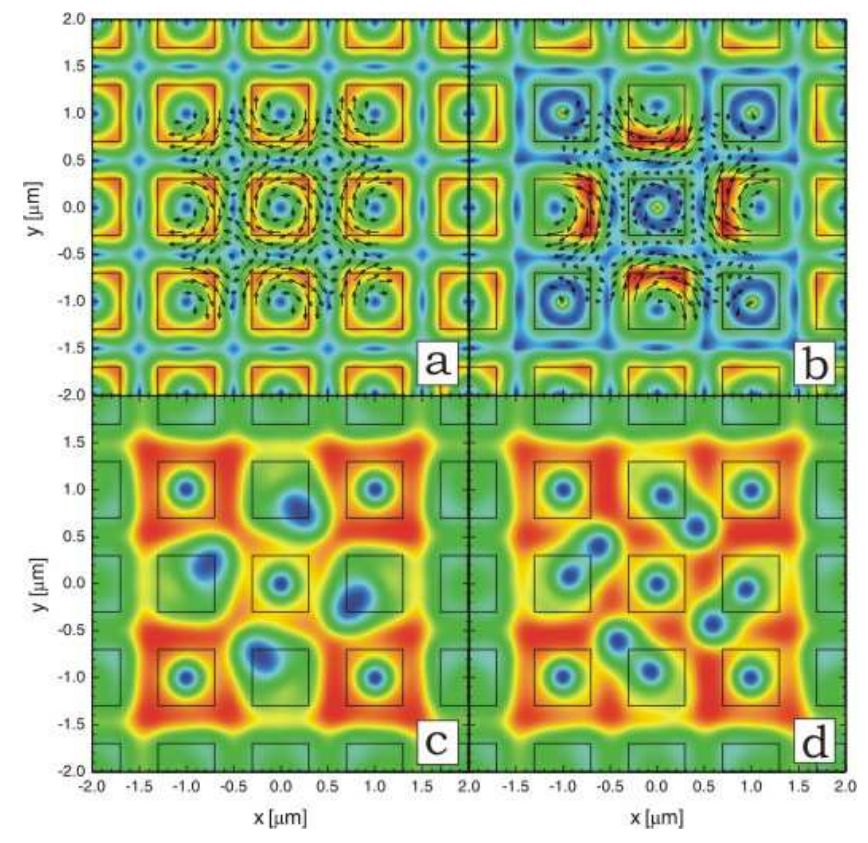

FIG. 5: The superfluid velocity contourplots $\left(v_{\max }=v_{c r}\right)$ and the corresponding vectorplots for $M=400 \mathrm{G}$ (see Fig. 4) and: (a) no applied field, (b) $H_{\text {ext }}=0.646 \mathrm{mT}$, just before the VAV nucleation. (c-d) the snapshots of the subsequent VAV nucleation process $\left(|\psi|^{2}\right.$ contourplots).

erned by the supervelocity $\boldsymbol{v} \sim \boldsymbol{j} /|\psi|^{2}$, plotted in Fig. 5 (a-b), for $M=400 \mathrm{G}$ without and with applied homogeneous field. For plain SCs in external homogeneous field, the vortex nucleation occurs for $v_{c r} \approx 1$, but for a VAV pair induced by a magnet this value is roughly $50 \%$ larger [note the non-quantized $M_{c r}$ (i.e. $\Phi^{+}$) in Fig. 2 and depends on the magnet parameters. The $\boldsymbol{v}$ vectorplot in Fig. 5(a) shows that MDs induce antivortex-like supercurrents, which compensate each other in interstitial areas and are maximal under the MD-edges. As explained above, for $H_{e x t}=0.646 \mathrm{mT}$, bringing 5 flux quanta in the region depicted in Fig. 5 added vortices are pinned by MDs in a regular formation. Therefore, at the pinning sites, MD-induced currents are compensated by the vortex currents (see Fig. 5(b)), which lowers the total energy and explains phenomenon (i). This current compensation also causes enhanced superconductivity in SC/FM samples when exposed to additional field (e.g. higher $M_{c r}$ in Fig. 4), contrary to conventional behavior, and explains the recent experimental findings of field-induced superconductivity 14]. On the other hand, the currents under MDs neighboring to the pinning sites are enhanced. As shown in (b), critical supervelocity $v_{c r}=1.447 H_{c 2} \xi$ is reached, and VAV pairs may nucleate. More importantly, the compensation of currents at the pinning sites leads to larger effective interstitial space for the nucleated antivortices. Therefore, contrary to the case in the absence of $H_{\text {ext }}$, there are no obstacles for VAV nucleation, and Figs. 5 (c-d) show snapshots of that process, on the path

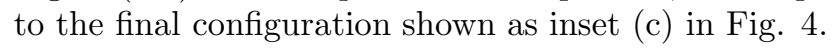

In conclusion, we described two novel scenarios of vortex-antivortex (VAV) nucleation in a SC film covered by magnetic dot (MD) arrays. The inhomogeneous stray field of the dots stimulates the appearance of VAV pairs. However, even when conditions for VAV nucleation are not fulfilled, the creation of these fascinating structures can be driven either by temperature variations, or applying a homogeneous magnetic field. Changing the temperature leads to a Little-Parks-like M-T phase boundary, where each cusp corresponds to the creation of a new VAV-pair per MD. Although counterintuitive, the applied homogeneous magnetic field embraces the creation of VAV-pairs, in cases when proximity of the magnets (compared to $\xi(\mathrm{T})$ ) does not support it. Pinned vortices at given MDs contribute to the critical nucleation conditions under the neighboring MDs in the lattice through the supercurrent compensation. As a result, a new type of VAV lattice is formed, with positive net vorticity.

The authors acknowledge support from the Flemish Science Foundation (FWO-Vl), the Belgian Science Policy, and the ESF programme "VORTEX".

* Electronic address: Francois.Peeters@ua.ac.be

[1] J. Schwinger, Phys. Rev. 82, 664 (1951).

[2] V.A. Schweigert and F.M. Peeters, Phys. Rev. B 51, 7700 (1995); A. Piel et al., Phys. Rev. Lett. 89, 085004 (2002).

[3] J.-P. Martikainen et al., Phys. Rev. A 64, 063602 (2001).

[4] T. Obata and M. Kubota, Phys. Rev. B 66, 140506(R) (2002).

[5] M.R. Beasley et al., Phys. Rev. Lett. 42, 1165 (1979).

[6] M.V. Fistul et al., Phys. Rev. Lett. 91, 257004 (2003).

[7] V.L. Berezinskii, Zh. Exp. Theor. Fiz. 59, 907 (1970); J.M. Kosterlitz and D.J. Thouless, J. Phys. C: Solid State Phys. 6, 1181 (1973).

[8] A.M. Kadin et al., Phys. Rev Lett. 65, 3193 (1990).

[9] L.F. Chibotaru et al., Nature (London) 408, 833 (2000).

[10] B.J. Baelus and F.M. Peeters, Phys. Rev. B 65, 104515 (2002); V.R. Misko et al., Phys. Rev. Lett. 90, 147003 (2003).

[11] D.J. Priour, Jr. and H.A. Fertig, Phys. Rev. Lett. 93, 057003 (2004).

[12] M.V. Milošević and F.M. Peeters, Phys. Rev. Lett. 93, 267006 (2004); ibid. Phys. Rev. B 68, 024509 (2003).

[13] J.I. Martin et al., Phys. Rev. Lett. 79, 1929 (1997); M.J. Van Bael et al., Phys. Rev. B 59, 14674 (1999); A.N. Grigorenko et al., Phys. Rev. Lett. 90, 237001 (2003).

[14] M. Lange et al., Phys. Rev. Lett. 90, 197006 (2003).

[15] R. Kato et al., Phys. Rev. B 47, 8016 (1993).

[16] L.P. Gor'kov and N.B. Kopnin, Sov. Phys. Usp. 18, 496 (1975).

[17] W.A. Little and R.D. Parks, Phys. Rev. Lett. 9, 9 (1962). 